\title{
S1 Protection
}

National Cancer Institute

\section{Source}

National Cancer Institute. S1 Protection. NCI Thesaurus. Code C19241.

Molecular biology procedure used to determine the 5' end or 3 ' end of a nucleic acid molecule, or the location of the junction between spliced molecules. Used most commonly to determine the 5 ' end of RNA transcripts. Based on the protection afforded an end-labeled DNA probe from nuclease S1 digestion after hybridization with a target molecule. The length of the protected probe indicates the end or molecular junction of the target nucleic acid sequence. 